\title{
Simpleness of Leavitt Path Algebras with Coefficients in a Commutative Semiring
}

\author{
Y. Katsov' ${ }^{1}$, T. G. Nam², J. Zumbrägel ${ }^{3}$ \\ katsov@hanover.edu; tgnam@math.ac.vn; jens.zumbragel@ucd.ie \\ ${ }^{1}$ Department of Mathematics \\ Hanover College, Hanover, IN 47243-0890, USA \\ ${ }^{2}$ Institute of Mathematics, VAST \\ 18 Hoang Quoc Viet, Cau Giay, Hanoi, Vietnam \\ ${ }^{3}$ Institute of Algebra \\ Dresden University of Technology, Germany
}

\begin{abstract}
In this paper, we study ideal- and congruence-simpleness for the Leavitt path algebras of directed graphs with coefficients in a commutative semiring $S$, as well as establish some fundamental properties of those algebras. We provide a complete characterization of idealsimple Leavitt path algebras with coefficients in a semifield $S$ that extends the well-known characterizations when the ground semiring $S$ is a field. Also, extending the well-known characterizations when $S$ is a field or commutative ring, we present a complete characterization of congruence-simple Leavitt path algebras over row-finite graphs with coefficients in a commutative semiring $S$.
\end{abstract}

Mathematics Subject Classifications: 16Y60, 16D99, 16G99, 06A12; 16S10, 16S34.

Key words: Congruence-simple and ideal-simple semirings, Leavitt path algebra.

\section{Introduction}

In some way, "prehistorical" beginning of Leavitt path algebras started with Leavitt algebras ([18] and [19]), Bergman algebras ([7]), and graph C*algebras $([9])$, considering rings with the Invariant Basis Number property,

The second author is supported by the Vietnam National Foundation for Science and Technology Development (NAFOSTED). The third author is supported by the Irish Research Council under Research Grant ELEVATEPD/2013/82. 
universal ring constructions, and the structure of a separable simple infinite $\mathrm{C}^{*}$-algebra, respectively. As to the algebraic structures known as Leavitt path algebras themselves, they were initiated and developed independently, and using different approaches, in the foundational papers on the subject [1] and [4]. Then, during the last decade, these algebras have continuously been of significant interest to mathematicians from different areas of mathematics such as ring and group theorists, analysts working in $\mathrm{C}^{*}$-algebras, and symbolic dynamicists, for example. For a detailed history and overview of the Leavitt path algebras we refer our potential readers to a recent quite remarkable and motivating survey on the subject [3].

In our time, we may clearly observe a steadily growing interest in developing algebraic and homological theories of semirings and semimodules, as well as in their numerous connections with, and applications in, different branches of mathematics, computer science, cryptography, quantum physics, and many other areas of science (see, e.g., [10]). As is well known, structure theories for varieties of algebras constitute an important "classical" area of the sustained interest in algebraic research. In those theories, socalled simple algebras, i.e., algebras possessing only two trivial congruences - the identity and universal ones - play a very important role of "building blocks." In addition, simple semirings, constituting another booming area of semiring research, have quite interesting and promising applications in various fields, in particular in cryptography (see, e.g., [20]). However, in contrast to the varieties of groups and rings, research on simple semirings has been started only recently, and therefore not much on the subject is known. Also, investigating semirings and their representations, one should undoubtedly use methods and techniques of both ring and lattice theory as well as diverse techniques and methods of categorical and universal algebra, and work in a "nonabelian environment." Perhaps all these circumstances explain why research on simple semirings is still behind of that for rings and groups (for some recent activity and results on this subject one may consult [21], [5], [22], [6], [25], [14], [15], [17], [16]).

Motivated by [3], in this paper we initiate a study of Leavitt path algebras (it deserves to be mentioned that, in some way, a generalization of an idea of Leavitt algebras from [18] to a semiring setting was earlier considered in [13]) in a nonadditive/nonabelian semiring setting — working with semirings and semimodules, we live in a "world without subtraction" and, therefore, have no privilege of the classical well developed techniques of additive/abelian categories of modules over rings. More precisely, we consider the concepts of Leavitt path algebras with coefficients in a commutative semiring $S$, and of ideal- and congruence-simpleness for those algebras; note that in our semiring setting, in contrast to the "additive" ring case, these 
two notions of simpleness are not the same (see, e.g., [16, Examples 3.8]) and should be differed. In light of this, presenting some new, important and interesting in our view, considerations, results and techniques regarding characterizations of ideal- and congruence-simple Leavitt path algebras over a commutative ground semiring $S$, extending the "classical" ring characterizations (see, [1, Theorem 3.11], [2, Theorem 3.1], [23, Theorem 6.18] and [12, Theorem 3.11]), as well as motivating an interest to this direction of research, is a main goal of our paper.

For the reader's convenience, all subsequently necessary basic concepts and facts on semirings and Leavitt path algebras with coefficients in a commutative semiring are collected in Section 2.

In Section 3, together with establishing some important properties of the Leavitt path algebras with coefficients in a commutative semiring $S$, we provide a complete characterization of ideal-simple Leavitt path algebras with coefficients in a semifield $S$ (Theorem 3.4), constituting one of the central results of the paper and extending the well-known characterizations (see, [1, Theorem 3.11], [2, Theorem 3.1], [23, Theorem 6.18] and [12, Theorem 3.11]) when the ground semiring $S$ is a field.

In Section 4, together with establishing some fundamental facts about the Leavitt path algebras with coefficients in the Boolean semifield $\mathbf{B}$ and combining them with Theorem 3.4, we present a complete characterization of congruence-simple Leavitt path algebras over row-finite graphs with coefficients in a commutative semiring $S$ (Theorem 4.5), constituting another main result of the paper and extending the well-known characterizations from [op. cit.]. It should be emphasized that, in contrast to the "classical" case of the ground structure $S$ to be a commutative ring, in order to establish these results in our semiring setting, one needs to exploit some innovative approach and techniques of universal algebra based on dealing with congruences rather then with ideals. Also, resolving [15, Problem 2] in the class of Leavitt path algebras with coefficients in a commutative semiring $S$, we show (Corollary 4.2) that for algebras of this class the congruence-simpleness implies their ideal-simpleness.

Finally, for notions and facts from semiring theory, we refer to [11].

\section{Basic concepts}

\subsection{Preliminaries on semirings}

Recall [11] that a hemiring is an algebra $(S,+, \cdot, 0)$ such that the following conditions are satisfied:

(1) $(S,+, 0)$ is a commutative monoid with identity element 0 ; 
(2) $(S, \cdot)$ is a semigroup;

(3) Multiplication distributes over addition from either side;

(4) $0 s=0=s 0$ for all $s \in S$.

A hemiring $S$ is commutative if $(S, \cdot)$ is a commutative semigroup; and a hemiring $S$ is additively idempotent if $a+a=a$ for all $a \in S$. Moreover, a hemiring $S$ is a semiring if its multiplicative semigroup $(S, \cdot)$ actually is a monoid $(S, \cdot, 1)$ with identity element 1 . A commutative semiring $S$ is a semifield if $(S \backslash\{0\}, \cdot, 1)$ is a group. Two well-known examples of semifields are the additively idempotent two element semiring $\mathbf{B}=\{0,1\}$, the so-called Boolean semifield, and the tropical semifield $(\mathbb{R} \cup\{-\infty\}, \vee,+,-\infty, 0\})$.

As usual, given two hemirings $S$ and $S^{\prime}$, a map $\varphi: S \longrightarrow S^{\prime}$ is a homomorphism iff $\varphi(x+y)=\varphi(x)+\varphi(y)$ for all $x, y \in S$; and a submonoid $I$ of $(S,+, 0)$ is an ideal of a hemiring $S$ iff $s a$ and $a s \in I$ for all $a \in I$ and $s \in S$; an equivalence relation $\rho$ on a hemiring $S$ is a congruence iff $(s+a, s+b) \in \rho v,(s a, s b) \in \rho$ and $(a s, b s) \in \rho$ for all pairs $(a, b) \in \rho$ and $s \in$ $S$. On every hemiring $S$ there are always the two trivial congruences - the diagonal congruence, $\triangle_{S}:=\{(s, s) \mid s \in S\}$, and the universal congruence, $S^{2}:=\{(a, b) \mid a, b \in S\}$. Following [5], a hemiring $S$ is congruence-simple if $\triangle_{S}$ and $S^{2}$ are the only congruences on $S$; and $S$ is ideal-simple if 0 and $S$ are the only ideals of $S$. It is clear that a hemiring $S$ is congruence-simple iff every nonzero hemiring homomorphism $\varphi: S \longrightarrow S^{\prime}$ is injective. Obviously, the concepts congruence- and ideal-simpleness are the same for rings and, therefore, we have just simple rings, but they are different ones for semirings in general (see, e.g., [16, Examples 3.8]).

An $S$-semimodule over a given commutative semiring $S$ is a commutative monoid $\left(M,+, 0_{M}\right)$ together with a scalar multiplication $(s, m) \mapsto s m$ from $S \times M$ to $M$ which satisfies the identities $\left(s s^{\prime}\right) m=s\left(s^{\prime} m\right), s\left(m+m^{\prime}\right)=$ $s m+s m^{\prime},\left(s+s^{\prime}\right) m=s m+s^{\prime} m, 1 m=m, s 0_{M}=0_{M}=0 m$ for all $s, s^{\prime} \in S$ and $m, m^{\prime} \in M$. Homomorphisms between semimodules and free semimodules are defined in the standard manner.

By an $S$-algebra $A$ over a given commutative semiring $S$ we mean an $S$ semimodule $A$ with an associative bilinear $S$-semimodule multiplication "." on $A$. An $S$-algebra $A$ is unital if $(A, \cdot)$ is actually a monoid with a neutral element $1_{A} \in A$, i.e., $a 1_{A}=a=1_{A} a$ for all $a \in A$. For example, every hemiring is an $\mathbb{N}$-algebra, where $\mathbb{N}$ is the semiring of the natural numbers with added 0; and, of course, every additively idempotent hemiring is a B-algebra.

Let $S$ be a commutative semiring and $\left\{x_{i} \mid i \in I\right\}$ a set of independent, noncommuting indeterminates. Then $S\left\langle x_{i} \mid i \in I\right\rangle$ will denote the free $S$ algebra generated by the indeterminates $\left\{x_{i} \mid i \in I\right\}$, whose elements are polynomials in the noncommuting variables $\left\{x_{i} \mid i \in I\right\}$ with coefficients 
from $S$ that commute with each variable $x_{i}, i \in I$.

Finally, let $S$ be a commutative semiring and $(G, \cdot, 1)$ a group. Then we can form the group semiring $S[G]$, whose elements are formal sums $\sum_{g \in G} a_{g} g$ with the coefficients $a_{g} \in S$ and the finite support, i.e., almost all $a_{g}=0$. As usual, the operations of addition and multiplication on $S[G]$ are defined as follows

$$
\begin{gathered}
\sum_{g \in G} a_{g} g+\sum_{g \in G} b_{g} g=\sum_{g \in G}\left(a_{g}+b_{g}\right) g, \\
\left(\sum_{g \in G} a_{g} g\right)\left(\sum_{h \in G} b_{h} h\right)=\sum_{t \in G} c_{t} t
\end{gathered}
$$

where $c_{t}=\sum a_{g} b_{h}$, with summation over all $(g, h) \in G \times G$ such that $g h=t$. Clearly, the elements of $S:=S \cdot 1$ commute with the elements of $G:=1 \cdot G$ under the multiplication in $S[G]$. In particular, one may easily see that $S[\mathbb{Z}] \cong S\left[x, x^{-1}\right]$, where $S\left[x, x^{-1}\right]$ is the algebra of the Laurent polynomials over $S$.

\subsection{Basics on Leavitt path algebras with coefficients in a commutative semiring}

In this subsection, we introduce Leavitt path algebras having coefficients in an arbitrary commutative semiring $S$. The construction of such algebras is, certainly, a straightforward generalization of the constructions of the Leavitt path algebras with the semiring $S$ to be a field and a commutative ring with unit originated in [1] and [24], respectively. All these constructions are crucially based on some general notions of graph theory that for the reader's convenience we reproduce here.

A (directed) graph $\Gamma=(V, E, s, r)$ consists of two disjoint sets $V$ and $E$ - vertices and edges, respectively - and two maps $s, r: E \longrightarrow V$. If $e \in E$, then $s(e)$ and $r(e)$ are called the source and range of $e$, respectively. The graph $\Gamma$ is row-finite if $\left|s^{-1}(v)\right|<\infty$ for every $v \in V$. A vertex $v$ for which $s^{-1}(v)$ is empty is called a sink; and a vertex $v$ is regular iff $0<\left|s^{-1}(v)\right|<$ $\infty$. A path $p=e_{1} \ldots e_{n}$ in a graph $\Gamma$ is a sequence of edges $e_{1}, \ldots, e_{n}$ such that $r\left(e_{i}\right)=s\left(e_{i+1}\right)$ for $i=1, \ldots, n-1$. In this case, we say that the path $p$ starts at the vertex $s(p):=s\left(e_{1}\right)$ and ends at the vertex $r(p):=r\left(e_{n}\right)$, and has length $|p|:=n$. We consider the vertices in $V$ to be paths of length 0 . If $s(p)=r(p)$, then $p$ is a closed path based at $v=s(p)=r(p)$. Denote by $\mathrm{CP}(v)$ the set of all such paths. A closed path based at $v, p=e_{1} \ldots e_{n}$, is a closed simple path based at $v$ if $s\left(e_{i}\right) \neq v$ for every $i>1$. Denote by $\operatorname{CSP}(v)$ the set of all such paths. If $p=e_{1} \ldots e_{n}$ is a closed path and all vertices $s\left(e_{1}\right), \ldots, s\left(e_{n}\right)$ are distinct, then the subgraph $\left(s\left(e_{1}\right), \ldots, s\left(e_{n}\right) ; e_{1}, \ldots, e_{n}\right)$ 
of the graph $\Gamma$ is called a cycle. An edge $f$ is an exit for a path $p=e_{1} \ldots e_{n}$ if $s(f)=s\left(e_{i}\right)$ but $f \neq e_{i}$ for some $1 \leq i \leq n$.

Definition 2.1 ( $c f$. [1, Definition 1.3] and [24, Definition 2.4]). Let $\Gamma=$ $(V, E, s, r)$ be a graph and $S$ a commutative semiring. The Leavitt path algebra $L_{S}(\Gamma)$ of the graph $\Gamma$ with coefficients in $S$ is the $S$-algebra presented by the set of generators $V \cup E \cup E^{*}$ - where $E \rightarrow E^{*}, e \mapsto e^{*}$, is a bijection with $V, E, E^{*}$ pairwise disjoint - satisfying the following relations:

(1) $v v^{\prime}=\delta_{v, v^{\prime}} v$ for all $v, v^{\prime} \in V$;

(2) $s(e) e=e=e r(e), r(e) e^{*}=e^{*}=e^{*} s(e)$ for all $e \in E$;

(3) $e^{*} f=\delta_{e, f} r(e)$ for all $e, f \in E$;

(4) $v=\sum_{e \in s^{-1}(v)} e e^{*}$ whenever $v \in V$ is a regular vertex.

It is easy to see that the mappings given by $v \mapsto v$, for $v \in V$, and $e \longmapsto e^{*}, e^{*} \longmapsto e$ for $e \in E$, produce an involution on the algebra $L_{S}(\Gamma)$, and for any path $p=e_{1} \ldots e_{n}$ there exists $p^{*}:=e_{n}^{*} \ldots e_{1}^{*}$.

Observe that the Leavitt path algebra $L_{S}(\Gamma)$ can also be defined as the quotient of the free $S$-algebra $S\left\langle v, e, e^{*} \mid v \in V, e \in E, e^{*} \in E^{*}\right\rangle$ by the congruence $\sim$ generated by the following ordered pairs:

(1) $\left(v v^{\prime}, \delta_{v, v^{\prime}} v\right)$ for all $v, v^{\prime} \in V$,

(2) $(s(e) e, e),(e, e r(e))$ and $\left(r(e) e^{*}, e^{*}\right),\left(e^{*}, e^{*} s(e)\right)$ for all $e \in E$,

(3) $\left(e^{*} f, \delta_{e, f} r(e)\right)$ for all $e, f \in E$,

(4) $\left(v, \sum_{e \in s^{-1}(v)} e e^{*}\right)$ for all regular vertices $v \in V$.

Remark 2.2. As will be shown in Proposition 2.4, for any graph $\Gamma=$ $(V, E, s, r)$, all generators $\left\{v, e, e^{*} \mid v \in V, e \in E, e^{*} \in E^{*}\right\}$ of $L_{S}(\Gamma)$ are nonzero. Furthermore, from the observation above, it readily follows that $L_{S}(\Gamma)$ is, in fact, the "largest" algebra generated by the elements $\left\{v, e, e^{*} \mid\right.$ $\left.v \in V, e \in E, e^{*} \in E^{*}\right\}$ satisfying the relations (1) - (4) of Definition 2.1, in other words, $L_{S}(\Gamma)$ has the following universal property: If $A$ is an $S$ algebra generated by a family of elements $\left\{a_{v}, b_{e}, c_{e^{*}} \mid c \in V, e \in E, e^{*} \in E^{*}\right\}$ satisfying the analogous to $(1)$ - (4) relations in Definition 2.1, then there always exists an $S$-algebra homomorphism $\varphi: L_{S}(\Gamma) \rightarrow A$ given by $\varphi(v)=a_{v}$, $\varphi(e)=b_{e}$ and $\varphi\left(e^{*}\right)=c_{e^{*}}$.

The following examples illustrate that some well-known (classical) algebras actually can be viewed as the Leavitt path algebras as well.

Examples 2.3 ( $c f$. [1, Examples 1.4]). Let $S$ be a commutative semiring.

(i) Let $\Gamma=(V, E, s, r)$ be a graph with $V=\left\{v_{1}, \ldots, v_{n}\right\}$ and $E=$ $\left\{e_{1}, \ldots, e_{n-1}\right\}$, where $s\left(e_{i}\right)=v_{i}, r\left(e_{i}\right)=v_{i+1}$ for all $i=1, \ldots, n-1$. Then it is easy to check that the map $\varphi: L_{S}(\Gamma) \longrightarrow M_{n}(S)$, given by $\varphi\left(v_{i}\right)=E_{i, i}$, $\varphi\left(e_{i}\right)=E_{i, i+1}$ and $\varphi\left(e_{i}^{*}\right)=E_{i+1, i}$, where $\left\{E_{i, j} \mid 1 \leq i, j \leq n\right\}$ are the 
standard elementary matrices in the $n \times n$ matrix semiring $M_{n}(S)$, is an $S$-algebra isomorphism.

(ii) Let $\Gamma=(V, E, s, r)$ be a graph given by $V=\{v\}$ and $E=\{e\}$. Then it is obvious that the Leavitt path algebra $L_{S}(\Gamma)$ is isomorphic to the Laurent polynomial algebra $S\left[x, x^{-1}\right]$ with $x:=e$ and $x^{-1}:=e^{*}$.

(iii) In [19], investigating rings with the Invariant Basis Number property there were introduced what we now call the Leavitt algebras of the form $L_{K}(1, n)$, where $K$ is a field and $n \geq 2$ is a natural number, of type $(1, n)$. Then, in [13], the authors, generalizing the Leavitt algebra construction in a semiring setting, constructed an $S$-algebra $L_{S}(1, n)$ over a commutative semiring $S$ which was defined by the generators $\left\{x_{i}, y_{i} \mid 1 \leq i \leq n\right\}$ and relations $x_{i} y_{j}=\delta_{i j}$ for all $1 \leq i, j \leq n$, and $\sum_{i=1}^{n} y_{i} x_{i}=1$. Considering the graph $\Gamma=(V, E, s, r)$ given by $V=\{v\}$ and $E=\left\{e_{1}, \ldots, e_{n}\right\}$, one may easily verify that the Leavitt path algebra $L_{S}(\Gamma)$ is, in fact, isomorphic to $L_{S}(1, n)$ by letting $y_{i}:=e_{i}$ and $x_{i}:=e_{i}^{*}$ for all $1 \leq i \leq n$.

The following proposition is an analog of [24, Proposition 3.4] for a nonabelian semiring setting and presents some fundamental properties of the Leavitt path algebras.

Proposition 2.4 (cf. [24, Proposition 3.4]). Let $\Gamma=(V, E, s, r)$ be a graph and $S$ a commutative semiring. Then, the Leavitt path algebra $L_{S}(\Gamma)$ has the following properties:

(1) All elements of the set $\left\{v, e, e^{*} \mid v \in V, e \in E, e^{*} \in E^{*}\right\}$ are nonzero;

(2) If $a, b$ are distinct elements in $S$, then $a v \neq b v$ for all $v \in V$;

(3) Every monomial in $L_{S}(\Gamma)$ is of the form $\lambda p q^{*}$, where $\lambda \in S$ and $p, q$ are paths in $\Gamma$ such that $r(p)=r(q)$.

Proof. The proof given for the case of rings in [24, Proposition 3.4], which, in turn, uses a similar construction as for the case of fields from [12, Lemma 1.5], is based on Remark 2.2 - there should be constructed an $S$-algebra $A$ as in Remark 2.2 having all generators $\left\{a_{v}, b_{e}, c_{e^{*}} \mid v \in V, e \in E, e^{*} \in E^{*}\right\}$ to be nonzero. It almost does not depend on the "abelianness" of the ring case and, therefore, it works in our semiring setting as well. Just for the reader's convenience, we have decided to sketch it here.

Thus, let $I$ be an infinite set of the cardinality at least $|V \cup E|$, and let $Z:=S^{(I)}$ a free $S$-semimodule with the basis $I$, i.e., $Z$ is a direct sum of $|I|$ copies of $S$. For each $e \in E$, let $A_{e}:=Z$ and, for each $v \in V$, let

$$
A_{v}:= \begin{cases}\bigoplus_{s(e)=v} A_{e} & \text { if }\left|s^{-1}(v)\right| \neq \varnothing \\ Z & \text { if } v \text { is a sink. }\end{cases}
$$

Note that all $A_{e}$ and $A_{v}$ are all mutually isomorphic, since each of them is the direct sum of $|I|$ many copies of $S$. Let $A:=\bigoplus_{v \in V} A_{v}$. For each 
$v \in V$ define $T_{v}: A_{v} \longrightarrow A_{v}$ to be the identity map and extend it to a homomorphism $T_{v}: A \longrightarrow A$ by defining $T_{v}$ to be zero on $A \ominus A_{v}$. Also, for each $e \in V$ choose an isomorphism $T_{e}: A_{r(e)} \longrightarrow A_{e} \subseteq A_{s(e)}$ and extend it to a homomorphism $T_{e}: A \longrightarrow A$ by mapping to zero on $A \ominus A_{r(e)}$. Finally, we define $T_{e^{*}}: A \longrightarrow A$ by taking the isomorphism $T_{e}^{-1}: A_{e} \subseteq A_{s(e)} \longrightarrow A_{r(e)}$ and extending it to a homomorphism $T_{e^{*}}: A \longrightarrow A$ by letting $T_{e^{*}}$ to be zero on $A \ominus A_{e}$.

Now consider the subalgebra of $\operatorname{Hom}_{S}(A, A)$ generated by $\left\{T_{v}, T_{e}, T_{e^{*}} \mid\right.$ $\left.v \in V, e \in E, e^{*} \in E^{*}\right\}$. It is straightforward to check (cf. [12, Lemma 1.5]) that $\left\{T_{v}, T_{e}, T_{e^{*}} \mid v \in V, e \in E, e^{*} \in E^{*}\right\}$ is a collection of nonzero elements satisfying the relations described in Definition 2.1. By the universal property of $L_{S}(\Gamma)$, we get that the elements of the set $\left\{v, e, e^{*}\right.$ | $\left.v \in V, e \in E, e^{*} \in E^{*}\right\}$ are nonzero and (1) is established.

Next we note that for each $v \in V$ we have $A_{v}=S \oplus M$ for some $S$-semimodule $M$. Let $a, b$ be two distinct elements in $S$. We have

$$
a T_{v}(1,0)=T_{v}(a, 0)=(a, 0) \neq(b, 0)=T_{v}(b, 0)=b T_{v}(1,0),
$$

so $a T_{v} \neq b T_{v}$. The universal property of $L_{S}(\Gamma)$ then implies that $a v \neq b v$, and (2) is established.

As to (3), it follows immediately from the fact that $e^{*} f=\delta_{e, f} r(e)$ for all $e, f \in E$.

As usual, for a hemiring $S$ a set of local units $F$ is a set $F \subseteq S$ of idempotents in $S$ such that, for every finite subset $\left\{s_{1}, \ldots, s_{n}\right\} \subseteq S$, there exists an element $f \in F$ with $f s_{i}=s_{i}=s_{i} f$ for all $1 \leq i \leq n$. Using Proposition 2.4 and repeating verbatim the proof of [1, Lemma 1.6], one obtains the following useful fact.

Proposition 2.5. Let $\Gamma=(V, E, s, r)$ be a graph and $S$ a commutative semiring. Then $L_{S}(\Gamma)$ is a unital $S$-algebra if $V$ is finite; and if $V$ is infinite, the set of all finite sums of distinct elements of $V$ is the set of local units of the $S$-algebra $L_{S}(\Gamma)$.

Let $\Gamma=(V, E, s, r)$ be a graph. A subset $H \subseteq V$ is called hereditary if $s(e) \in H$ implies $r(e) \in H$ for all $e \in E$; and $H \subseteq V$ is saturated if $v \in H$ for any regular vertex $\nu$ with $r\left(s^{-1}(v)\right) \subseteq H$. Obviously, the two trivial subsets of $V, \varnothing$ and $V$, are hereditary and saturated ones. We note the following useful observation whose proof is completely analogous to the ones in $[1$, Lemma 3.9] and [2, Lemma 2.3] and which, for the reader's convenience, we provide here.

Lemma 2.6. Let $\Gamma=(V, E, s, r)$ be a graph, $S$ a commutative semiring, and $I$ an ideal of $L_{S}(\Gamma)$. Then, $I \cap V$ is a hereditary and saturated subset of $V$. 
Proof. For any $e \in E$ with $s(e) \in H$ we have $r(e) \in H$, since $e=s(e) e \in I$, and thus $r(e)=e^{*} e \in I$. Furthermore, if a regular vertex $v \in V$ satisfies $r(e) \in H$ for all $e \in E$ with $s(e)=v$, then $v \in H$, since $e=\operatorname{er}(e) \in I$ for all these edges $e \in E$, and hence $v=\sum_{e \in s^{-1}(v)} e e^{*} \in I$.

We conclude this section with the following, although simple but quite useful, technical remark obviously following from the identity $e^{*} f=\delta_{e, f} r(e)$ for all $e, f \in E$.

Remark 2.7. For any two paths $p, q$ in $\Gamma$ we have

$$
p^{*} q= \begin{cases}q^{\prime} & \text { if } q=p q^{\prime} \\ r(p) & \text { if } p=q \\ p^{\prime *} & \text { if } p=q p^{\prime} \\ 0 & \text { otherwise }\end{cases}
$$

\section{Ideal-simpleness of Leavitt path algebras with coefficients in a semifield}

The main goal of this section is to present a description of the ideal-simple Leavitt path algebras $L_{S}(\Gamma)$ of arbitrary graphs $\Gamma=(V, E, s, r)$ with coefficients in a semifield $S$ that extends the well-known description when the ground semifield $S$ is a field $K$ ([1, Theorem 3.11], [2, Theorem 3.1], [23, Theorem 6.18] and [12, Theorem 3.11]). For that we have to establish some subsequently needed important facts.

Proposition 3.1. A graph $\Gamma=(V, E, s, r)$ of an ideal-simple Leavitt path algebra $L_{S}(\Gamma)$ with coefficients in a commutative semiring $S$ satisfies the following two conditions:

(1) The only hereditary and saturated subset of $V$ are $\varnothing$ and $V$;

(2) Every cycle in $\Gamma$ has an exit.

Proof. (1) Actually the proof of the statement given in [1, Theorem 3.11] does not use the additive ring/module setting and, therefore, it can be easily modified for our (nonadditive) semiring setting. For the reader's convenience, we briefly sketch central ideas of that modification here.

Assume that $V$ contains a nontrivial hereditary and saturated subset $H$. In the same way as was shown in [1, Theorem 3.11], one may easily observe that

$$
\Gamma^{\prime}=\left(V^{\prime}, E^{\prime}, r_{\Gamma^{\prime}}, s_{\Gamma^{\prime}}\right):=\left(V \backslash H, r^{-1}(V \backslash H),\left.r\right|_{V \backslash H},\left.s\right|_{V \backslash H}\right)
$$

is a graph, too. Then, as in [1, Theorem 3.11], let us consider an $S$-algebra homomorphism $\varphi: L_{S}(\Gamma) \longrightarrow L_{S}\left(\Gamma^{\prime}\right)$ given on the generators of the free 
$S$-algebra $A:=S\left\langle v, e, e^{*} \mid v \in V, e \in E, e^{*} \in E^{*}\right\rangle$ as follows: $\varphi(v)=$ $\chi_{V^{\prime}}(v) v, \varphi(e)=\chi_{E^{\prime}}(e) e$ and $\varphi\left(e^{*}\right)=\chi_{\left(E^{\prime}\right)^{*}}\left(e^{*}\right) e^{*}$, where $\chi_{X}$ denotes the usual characteristic function of a set $X$. To be sure that in a such manner defined map $\varphi: L_{S}(\Gamma) \longrightarrow L_{S}\left(\Gamma^{\prime}\right)$, indeed, provides us with the desired hemiring homomorphism, we only need to verify that all following pairs

$\left(v v^{\prime}, \delta_{v, v^{\prime}} v\right)$ for all $v, v^{\prime} \in V$

$(s(e) e, e),(e, e r(e))$ and $\left(r(e) e^{*}, e^{*}\right),\left(e^{*}, e^{*} s(e)\right)$ for all $e \in E$,

$\left(e^{*} f, \delta_{e, f} r(e)\right)$ for all $e, f \in E$,

$\left(v, \sum_{e \in s^{-1}(v)} e e^{*}\right)$ for a regular vertex $v \in V$,

are in the kernel congruence

$$
\operatorname{ker}(\varphi):=\left\{(x, y) \in A^{2} \mid \varphi(x)=\varphi(y)\right\}
$$

of $\varphi$. But the latter can be established right away by repeating verbatim the corresponding obvious arguments in the proof of [1, Theorem 3.11]. Note that $\left|\left(s_{\Gamma^{\prime}}\right)^{-1}(v)\right|<\infty$ in $\Gamma^{\prime}$ for any regular vertex $v$ in $\Gamma$. For $\varnothing \neq H \varsubsetneqq V$ and Proposition 2.4, $\varphi$ is a nonzero homomorphism and $H \subseteq \varphi^{-1}(0)$; and therefore, $L_{S}(\Gamma)$ contains a proper ideal and, hence, is not ideal-simple.

(2) Let $\Gamma$ contain a cycle $p$, based at $v$, without any exit. Then, by repeating verbatim the corresponding arguments in the proof of $[1$, Theorem 3.11], one gets that $v L_{S}(\Gamma) v=S\left[p, p^{*}\right]$, i.e., each element in $v L_{S}(\Gamma) v$ is written in the form $\sum_{i=r}^{s} \lambda_{i} p^{i}$, where $r, s \in \mathbb{Z}$ and $\lambda_{i} \in S$; and let $p^{0}:=v$ and $p^{-j}:=\left(p^{*}\right)^{j}$ for all $j>0$. For $L_{S}(\Gamma)$ is ideal-simple and [16, Proposition 5.3], $v L_{S}(\Gamma) v$ is an ideal-simple commutative semiring as well. The latter, by [5, Theorem 11.2], implies that $v L_{S}(\Gamma) v=S\left[p, p^{*}\right]$ is a semifield. We claim that $S\left[p, p^{*}\right] \cong S\left[x, x^{-1}\right]$, the Laurent polynomial semiring over $S$; as this is clearly not a semifield, this contradiction finishes the proof.

It remains to show that the natural homomorphism $S\left[x, x^{-1}\right] \rightarrow S\left[p, p^{*}\right]$ given by $f \mapsto f(p)$ is, indeed, injective. Let $I, Z=S^{(I)}, A_{e}$ for $e \in E, A_{v}$ for $v \in V$, and $A$ be as in the proof of Proposition 2.4, and consider the endomorphisms $T_{v}, T_{e}, T_{e^{*}}$ of $A$, for $v \in V, e \in E, e^{*} \in E^{*}$. Without loss of generality, we may assume that $I=\mathbb{Z} \times I^{\prime}$ for some nonempty set $I^{\prime}$.

Write the cycle $p$ based at $v$ as $p=e_{1} \ldots e_{n}$ with $e_{i} \in E$, where $v_{i-1}:=$ $s\left(e_{i}\right)$ and $v_{i}:=r\left(e_{i}\right)$ for $1 \leq i \leq n$, so that $v_{0}=v_{n}=v$. By the construction in the proof of Proposition 2.4 we have $A_{v_{i-1}}=A_{e_{i}}$, since $p$ has no exit, and $T_{e_{i}}$ restricts to an isomorphism $A_{v_{i}} \longrightarrow A_{v_{i-1}}$, for all $i$. Consider the endomorphism $T_{p}:=T_{e_{1}} \circ \cdots \circ T_{e_{n}}$, which restricts to an isomorphism $T: A_{v} \longrightarrow A_{v}$, where $A_{v}=Z=S^{(I)}=S^{\left(\mathbb{Z} \times I^{\prime}\right)}$. Observe that there is no limitation in choosing these isomorphisms, hence we may assume that $T\left(\delta_{(k, i)}\right)=\delta_{(k+1, i)}$ for all $k \in \mathbb{Z}$ and $i \in I^{\prime}$, where by $\delta_{(k, i)}$ we denote the standard basis vectors of the free $S$-semimodule $S^{\left(\mathbb{Z} \times I^{\prime}\right)}$. 
Now suppose that $f(p)=g(p)$ for some Laurent polynomials $f, g \in$ $S\left[x, x^{-1}\right]$. Since the $T_{v}, v \in V, T_{e}, e \in E, T_{e^{*}}, e^{*} \in E^{*}$, satisfy the relations described in Definition 2.1, it follows that $f(T)=g(T)$ holds in $\operatorname{Hom}_{S}(A, A)$. Writing $f=\sum_{j=r}^{s} f_{j} x^{j}$ and $g=\sum_{j=r}^{s} g_{j} x^{j}$ for some $r, s \in \mathbb{Z}$ and $f_{j}, g_{j} \in S$, from our choice of $T$ we see that $f(T)\left(\delta_{0, i}\right)=\sum_{j=r}^{s} f_{j} T^{j}\left(\delta_{0, i}\right)=\sum_{j=r}^{s} f_{j} \delta_{j, i}$, and similarly $g(T)\left(\delta_{0, i}\right)=\sum_{j=r}^{s} g_{j} \delta_{j, i}$, for any $i \in I^{\prime}$. From this we readily deduce that $f=g$ and thus the injectivity follows.

Following [1], a monomial in $L_{S}(\Gamma)$ is a real path if it contains no terms of the form $e^{*} \in E^{*}$, and a polynomial $\alpha \in L_{S}(\Gamma)$ is in only real edges if it is a sum of real paths; let $L_{S}(\Gamma)_{\text {real }}$ denote the subhemiring of all polynomials in only real edges in $L_{S}(\Gamma)$. The following technical observation will prove to be useful.

Lemma 3.2 (cf. [1, Corollary 3.2]). Let $\Gamma=(V, E, s, r)$ be a graph with the property that every cycle has an exit and $S$ a semifield. Then, if $\alpha \in$ $L_{S}(\Gamma)_{\text {real }} \subseteq L_{S}(\Gamma)$ is a nonzero polynomial in only real edges, then there exist $a, b \in L_{S}(\Gamma)$ such that $a \alpha b \in V$.

Proof. The proof of [1, Corollary 3.2] does not use the "additiveness" of the setting and, therefore, repeating verbatim the latter, one gets the statement in our nonadditive setting as well. However, we provide a new proof which is much shorter than the Abrams and Aranda Pino's original proof.

Namely, we write $\alpha$ in the form $\alpha=\sum_{i} \lambda_{i} q_{i}$ with $q_{i}$ distinct real paths and $0 \neq \lambda_{i} \in S$. Out of the set $\left\{q_{i}\right\}$ choose $p$ such that no proper prefix path of $p$ is contained therein. Let $v=r(p)$. Then, using Remark 2.7 we get $p^{*} \alpha v=\lambda v+\sum_{i} \lambda_{i} p^{*} q_{i}$, where the sum is over all $q_{i}$ that have $p$ as a proper prefix path and $r\left(q_{i}\right)=v$, so that $p^{*} q_{i} \in \mathrm{CP}(v)$.

Hence, without loss of generality, we may assume that $\alpha=\lambda v+\sum_{i=1}^{n} \lambda_{i} p_{i}$, where $p_{i} \in \operatorname{CP}(v)$ of positive length and $0 \neq \lambda \in S$. Fix some $c \in \operatorname{CSP}(v)$. For any $p_{i} \in \operatorname{CP}(v)$ we may write $p_{i}=c^{n_{i}} p_{i}^{\prime}$ with $n_{i} \in \mathbb{N}$ maximal, so that either $p_{i}^{\prime}=v$ or $p_{i}^{\prime}=d_{i} p_{i}^{\prime \prime}$ with $d_{i} \in \operatorname{CSP}(v), d_{i} \neq c$, in which case $\left(c^{*}\right)^{n_{i}+1} p_{i}=c^{*} p_{i}^{\prime}=c^{*} d_{i} p_{i}^{\prime \prime}=0$ by Remark 2.7. With $n:=\max \left\{n_{i} \mid i=\right.$ $1, \ldots, n\}+1$, we then have that $\left(c^{*}\right)^{n} p_{i} c^{n}=p_{i}$ if $p_{i}=c^{n_{i}}$, and $\left(c^{*}\right)^{n} p_{i} c^{n}=0$ otherwise. Therefore, we have $\alpha^{\prime}:=\left(c^{*}\right)^{n} \alpha c^{n}=\lambda v+\sum_{j} \lambda_{j} c^{n_{j}}$ with $n_{j}>0$, i.e., $\alpha^{\prime}=\lambda v+c P(c)$ for some polynomial $P$. Now, we write $c$ in the form $c=e_{1} \ldots e_{m}$. By our hypothesis and [1, Lemma 2.5], there exists an exit $f \in E$ for $c$, that is, there exists $j \in\{1, \ldots, m\}$ such that $s(f)=s\left(e_{j}\right)$ but $f \neq e_{j}$. Let $z:=e_{1} \ldots e_{j-1} f$. We get that $s(z)=v$ and $z^{*} c=0$, so that $\lambda^{-1} z^{*} \alpha^{\prime} z=z^{*} z+\lambda^{-1} z^{*} c P(c) z=r(z) \in V$, as desired.

As was shown in [8, Theorem 6], every nonzero ideal of the Leavitt path algebra of a row-finite graph with coefficients in a field always contains a 
nonzero polynomial in only real edges. The following observation extends this result to the Leavitt path algebra over an arbitrary graph.

Proposition 3.3. Let $\Gamma=(V, E, s, r)$ be a graph and $S$ a commutative semiring. Then any nonzero ideal I of $L_{S}(\Gamma)$ contains a nonzero polynomial in only real edges.

Proof. Let $I_{\text {real }}:=I \cap L_{S}(\Gamma)_{\text {real }}$ for a nonzero ideal $I$, and suppose that $I_{\text {real }}=0$. Choose $0 \neq \alpha=\sum_{i=1}^{d} \lambda_{i} p_{i} q_{i}^{*}$ in $I$, where $d$ is minimal such that $p_{1}, \ldots, p_{d}, q_{1}, \ldots, q_{d}$ are paths in $\Gamma$ and $0 \neq \lambda_{i} \in S, i=1, \ldots, d$. By using [8, Remark 3], as in the proof [8, Lemma 4], one can easily get that the element $\alpha$ can be presented in the form $\alpha=\mu_{1}+\cdots+\mu_{m}$, where all monomials in $\mu_{j} \in I, j=1, \ldots, m$, have the same source and the same range. Moreover, for $\alpha \neq 0$ and the minimality of $d$, we can assume that actually $\alpha=\sum_{i=1}^{d} \lambda_{i} p_{i} q_{i}^{*}$ with $s\left(p_{i}\right)=s\left(p_{j}\right)$ and $s\left(q_{i}\right)=s\left(q_{j}\right)=w \in V$ for all $i$ and $j$. Among all such $\alpha=\sum_{i=1}^{d} \lambda_{i} p_{i} q_{i}^{*} \in I$ with minimal $d$, select one for which $\left(\left|q_{1}\right|, \ldots,\left|q_{d}\right|\right)$ is the smallest in the lexicographic order of $\mathbb{N}^{d}$. Obviously, $\left|q_{i}\right|>0$ for some $i$ (otherwise, $0 \neq \alpha \in I_{\text {real }}=0$ ). If $e \in E$, then

$$
\alpha e=\sum_{i=1}^{d} \lambda_{i} p_{i} q_{i}^{*} e=\sum_{i=1}^{d^{\prime}} \lambda_{i} p_{i}^{\prime}\left(q_{i}^{\prime}\right)^{*}
$$

where we either have $d^{\prime}<d$, or $d^{\prime}=d$ and $\left(\left|q_{1}^{\prime}\right|, \ldots,\left|q_{d}^{\prime}\right|\right)$ is smaller than $\left(\left|q_{1}\right|, \ldots,\left|q_{d}\right|\right)$. Whence, for the minimality of $\left(\left|q_{1}\right|, \ldots,\left|q_{d}\right|\right)$, we get $\alpha e=0$ for all $e \in E$. For $\left|q_{i}\right|>0$ for some $i$, we have that $w$ is not a sink, and if it is a regular vertex, we have

$$
0 \neq \alpha=\alpha w=\alpha \sum_{e \in s^{-1}(w)} e e^{*}=\sum_{e \in s^{-1}(w)}(\alpha e) e^{*}=0 .
$$

Therefore, we need only to consider two possible cases when the vertex $w$ emits infinitely many edges:

Case 1. Let $\left|q_{j}\right|>0$ for all $j$, and $A:=\left\{e \in s^{-1}(w) \mid q_{i}^{*} e \neq 0\right.$ for some $1 \leq i \leq d\}$. Notice that $q_{i}^{*} e \neq 0$ if and only if the path $q_{i}$ has the form $q_{i}=f_{1} \ldots f_{k}$ with $k \geq 1$ and $f_{1}=e$. Specially, in this case, we have that $q_{i}^{*} e e^{*}=q_{i}^{*}$. It is clear that $|A|<\infty$, and hence, $\alpha=\sum_{e \in A} \alpha e e^{*}$. For $\alpha e=0$ for all $e \in E$, we have $0 \neq \alpha=\sum_{e \in A} \alpha e e^{*}=0$.

Case 2. If $\left|q_{j}\right|=0$ for some $j$, the element $\alpha$ can be presented as

$$
\alpha=\lambda_{1} p_{1}+\cdots+\lambda_{m} p_{m}+\lambda_{m+1} p_{m+1} q_{m+1}^{*}+\cdots+\lambda_{d} p_{d} q_{d}^{*},
$$

where $p_{1}, \ldots, p_{m}$ are distinct paths in $\Gamma$ and $r\left(p_{i}\right)=w=s\left(q_{j}\right)$ for all $i=1, \ldots, d$ and $j=m+1, \ldots, d$. Set $\beta:=\lambda_{1} p_{1}+\cdots+\lambda_{m} p_{m}$. By Remark 2.7, we may choose a path $p$ in $\Gamma$ such that

$$
p^{*} \beta=\lambda w+\sum_{j=1}^{k} \nu_{j} p_{j}^{\prime}
$$


where $0 \neq \lambda \in S, \nu_{j} \in S$ and $p_{j}^{\prime} \in \mathrm{CP}(w)$ for all $j$. For $w$ emits infinitely many edges, there is an edge $e \in s^{-1}(w)$ such that $q_{i}^{*} e=0=e^{*} p_{j}^{\prime}$ for all $i=m+1, \ldots, d$ and $j=1, \ldots, k$. Then, $0=\alpha e=\beta e \in I$ and, hence, $p^{*} \beta e=\lambda e+\sum_{j=1}^{k} \nu_{j} p_{j}^{\prime} e=0$. It implies that $e^{*} p^{*} \beta e=\lambda r(e)=0$. Using Proposition 2.4(2), we get that $\lambda=0$, a contradiction.

Hence, the ideal $I$ contains a nonzero polynomial in only real edges.

In [1, Theorem 3.11], the authors characterized the simple Leavitt path algebras over countable row-finite graphs with coefficients in a field. Then, the row-finiteness hypothesis independently was eliminated by the authors ([2, Theorem 3.1]) and in ([23, Theorem 6.18]), and finally this characterization has been extended in [12, Theorem 3.11] to arbitrary graphs. The next and main result of this section is an extension of the latter characterization to the Leavitt path algebras with coefficients in a semifield.

Theorem 3.4. A Leavitt path algebra $L_{S}(\Gamma)$ of a graph $\Gamma=(V, E, s, r)$ with coefficients in a semifield $S$ is ideal-simple if and only if the graph $\Gamma$ satisfies the following two conditions:

(1) The only hereditary and saturated subset of $V$ are $\varnothing$ and $V$;

(2) Every cycle in $\Gamma$ has an exit.

Proof. $\Longrightarrow$. It follows from Proposition 3.1.

$\Longleftarrow$. Let $I$ be a nonzero ideal of $L_{S}(\Gamma)$. By Proposition 3.3, I contains a nonzero polynomial $\alpha$ in only real edges. By Lemma 3.2, there exist $a, b \in L_{S}(\Gamma)$ such that $a \alpha b \in V$, i.e., $I \cap V \neq \varnothing$. Now, applying Lemma 2.6 and Proposition 2.5, we conclude that $I=L_{S}(\Gamma)$.

Taking into consideration [24, Theorem 7.20], the following question seems to be reasonable, interesting and promising.

Problem. How far can Theorem 3.4 be extended for the commutative ground semiring $S$ ?

We finish this section by demonstrating the use of Theorem 3.4 in reestablishing the ideal-simpleness of the Leavitt path algebras of Examples 2.3.

Examples 3.5 ( $c f$. [1, Corollary 3.13]). Note that all Leavitt path algebras in these examples are algebras with coefficients in a semifield $S$.

(i) By [15, Proposition 4.7], $M_{n}(S)$ is an ideal-simple algebra. However, this fact can also be justified by Theorem 3.4, since it is easy to check that the graph $\Gamma$ of Examples 2.3 (i) satisfies (1) and (2) of Theorem 3.4.

(ii) By Examples 2.3 (ii), the Laurent polynomial algebras $S\left[x, x^{-1}\right] \cong$ $L_{S}(\Gamma)$ where the graph $\Gamma$ contains a cycle without an exit, and therefore, by Theorem 3.4, $S\left[x, x^{-1}\right]$ is not ideal-simple. 
(iii) By Examples 2.3 (iii), the Leavitt algebras $L_{S}(1, n)$ for $n \geq 2$ are isomorphic to the Leavitt path algebras $L_{S}(\Gamma)$ such that for the graphs $\Gamma$ conditions (1) and (2) of Theorem 3.4 are obviously satisfied, and therefore, the algebras $L_{S}(1, n)$ are ideal-simple. (Note that we consider here an $S$ algebra analog of a Leavitt algebra over a field, see [19, Theorem 2]).

\section{Congruence-simpleness of Leavitt path al- gebras with coefficients in a commutative semiring}

Providing necessary and sufficient conditions for a Leavitt path algebra over a row-finite graph with coefficients in a commutative semiring to be congruence-simple is the main goal of this section. We start with necessary conditions for such algebras to be congruence-simple, namely:

Proposition 4.1. For a congruence-simple Leavitt path algebra $L_{S}(\Gamma)$ of a graph $\Gamma=(V, E, s, r)$ with coefficients in a commutative semiring $S$ the following statements are true:

(1) $S$ is either a field, or the Boolean semifield $\mathbf{B}$;

(2) The only hereditary and saturated subset of $V$ are $\varnothing$ and $V$;

(3) Every cycle in $\Gamma$ has an exit.

Proof. (1) First, let us show that there are only the two trivial congruences on $S$. Indeed, if $\sim$ is a proper congruence on $S$, the natural surjection $\pi: S \longrightarrow \bar{S}:=S / \sim$, defined by $\pi(\lambda)=\bar{\lambda}$, is neither zero nor an injective homomorphism. As one can easily verify, the homomorphism $\pi$ induces a nonzero surjective hemiring homomorphism $\varphi: L_{S}(\Gamma) \longrightarrow L_{\bar{S}}(\Gamma)$ such that $\varphi\left(\lambda p q^{*}\right)=\bar{\lambda} p q^{*}$, where $\lambda \in S$ and $p, q$ are paths in $\Gamma$ with $r(p)=r(q)$. For $\pi$ is not injective, there exist two distinct elements $a, b \in S$ such that $\bar{a}=\bar{b}$ and, by Proposition 2.4(2), $a v \neq b v$ in $L_{S}(\Gamma)$ for any $v \in V$. However,

$$
\varphi(a v)=\bar{a} v=\bar{b} v=\varphi(b v)
$$

and hence, $\varphi$ is not injective, and therefore, $L_{S}(\Gamma)$ is not congruence-simple. Thus, $S$ is congruence-simple, and it follows by [5, Theorem 10.1] (see also [21, Theorem 3.2]) that $S$ is either a field, or the semifield $\mathbf{B}$.

(2) A proof of this statement is established by the proof of Proposition $3.1(1)$ : Indeed, in the notation of the latter, one readily concludes that the $\operatorname{map} \varphi: L_{S}(\Gamma) \longrightarrow L_{S}\left(\Gamma^{\prime}\right)$ is a nonzero homomorphism and $H \subseteq \varphi^{-1}(0)$, and hence, $L_{S}(\Gamma)$ is not congruence-simple.

(3) This statement can be proven analogously to the proof of Proposition $3.1(2)$; and in the notations of the latter, one readily concludes that 
$v L_{S}(\Gamma) v=S\left[p, p^{*}\right] \cong S\left[x, x^{-1}\right]$, the Laurent polynomial semiring over $S$. By [16, Proposition $5.3(2)], v L_{S}(\Gamma) v$ is a congruence-simple semiring; that means, $S\left[x, x^{-1}\right]$ is congruence-simple, too. This would imply, by $[5$, Theorem 10.1] (see also [21, Theorem 3.2]), that $S\left[x, x^{-1}\right]$ is either a field or the Boolean semifield $\mathbf{B}$, what is obviously is not a case.

Combining Theorem 3.4 and Proposition 4.1, one immediately obtains that the congruence-simpleness of a Leavitt path algebra over an arbitrary graph with coefficients in a commutative semiring implies its idealsimpleness, what, in turn, actually resolves [15, Problem 2] in the class of Leavitt path algebras, namely:

Corollary 4.2. A congruence-simple Leavitt path algebra $L_{S}(\Gamma)$ over an arbitrary graph $\Gamma$ with coefficients in a commutative semiring $S$ is idealsimple as well.

Next, modifying the ideas and techniques used in the proof of $[8$, Theorem 6], we obtain a semiring version of this result for the Leavitt path algebras over the Boolean semifield $\mathbf{B}$.

Proposition 4.3. Let $\Gamma=(V, E, s, r)$ be a row-finite graph, $\rho$ a congruence on $L_{\mathbf{B}}(\Gamma)$, and $\rho_{\text {real }}:=\rho \cap\left(L_{\mathbf{B}}(\Gamma)_{\text {real }}\right)^{2}$. Then $\rho$ is generated by $\rho_{\text {real }}$.

Proof. Let $\tau$ be the congruence on $L_{\mathbf{B}}(\Gamma)$ generated by $\rho_{\text {real }}$, then the inclusion $\tau \subseteq \rho$ is obvious. Suppose that $\tau \neq \rho$, i.e., there exists $(x, y) \in \rho$ with $(x, y) \notin \tau$. By Proposition 2.5 we may choose a finite subset $F \subseteq V$ such that $x=x \sum_{v \in F} v$ and $y=y \sum_{v \in F} v$, and therefore

$$
(x, y)=\left(x \sum_{v \in F} v, y \sum_{v \in F} v\right)=\sum_{v \in F}(x v, y v)
$$

Since $(x, y) \notin \tau$, there exists $v \in F$ such that $(x v, y v) \notin \tau$, and we have $(x v, y v) \in \rho$. Therefore, we may assume that $x=\sum_{i=1}^{k} p_{i} q_{i}^{*}$ and $y=$ $\sum_{j=1}^{l} \gamma_{j} \delta_{j}^{*}$ with $p_{i}, q_{i}, \gamma_{j}, \delta_{j}$ paths in $\Gamma$ and $r\left(q_{i}^{*}\right)=r\left(\delta_{j}^{*}\right)=v$ for all $i, j$. Among all such pairs $\left(\sum_{i=1}^{k} p_{i} q_{i}^{*}, \sum_{i=1}^{l} \gamma_{i} \delta_{i}^{*}\right) \in \rho \backslash \tau$ with minimal $d:=$ $k+l$, select one for which $\left(\left|q_{1}\right|, \ldots,\left|q_{k}\right|,\left|\delta_{1}\right|, \ldots,\left|\delta_{l}\right|\right)$ is the smallest in the lexicographic order of $\mathbb{N}^{d}$. As $(x, y) \notin \tau$, one has $\left|q_{i}\right|>0$ for some $i$, or $\left|\delta_{j}\right|>0$ for some $j$. For all $e \in E$,

$$
(x e, y e)=\left(\sum_{i=1}^{k} p_{i} q_{i}^{*} e, \sum_{i=1}^{l} \gamma_{i} \delta_{i}^{*} e\right)=\left(\sum_{i=1}^{k^{\prime}} p_{i}^{\prime}\left(q_{i}^{\prime}\right)^{*}, \sum_{i=1}^{l^{\prime}} \gamma_{i}^{\prime}\left(\delta_{i}^{\prime}\right)^{*}\right)
$$

and either $d^{\prime}:=k^{\prime}+l^{\prime}<d$, or $d^{\prime}=d$ and $\left(\left|q_{1}^{\prime}\right|, \ldots,\left|q_{k}^{\prime}\right|,\left|\delta_{1}^{\prime}\right|, \ldots,\left|\delta_{l}^{\prime}\right|\right)$ is smaller than $\left(\left|q_{1}\right|, \ldots,\left|q_{k}\right|,\left|\delta_{1}\right|, \ldots,\left|\delta_{l}\right|\right)$, whence $(x e, y e) \in \tau$, by minimality. As some $\left|q_{i}\right|>0$ or some $\left|\delta_{j}\right|>0$, it follows that $v$ is not a 
sink, and hence, $(x, y)=(x v, y v)=\left(x \sum_{e \in s^{-1}(v)} e e^{*}, y \sum_{e \in s^{-1}(v)} e e^{*}\right)=$ $\sum_{e \in s^{-1}(v)}\left((x e) e^{*},(y e) e^{*}\right) \in \tau$, contradicting that $(x, y) \notin \tau$. This shows that $\rho=\tau$.

The following result, being an B-algebra analog of [1, Theorem 3.11], characterizes the congruence-simple Leavitt path algebras over the Boolean semifield B.

Theorem 4.4. A Leavitt path algebra $L_{\mathbf{B}}(\Gamma)$ of a row-finite graph $\Gamma=$ $(V, E, s, r)$ is congruence-simple if and only if the graph $\Gamma$ satisfies the following two conditions:

(1) The only hereditary and saturated subset of $V$ are $\varnothing$ and $V$;

(2) Every cycle in $\Gamma$ has an exit.

Proof. $\Longrightarrow$. It follows from Proposition 4.1.

$\Longleftarrow$. Let $\rho \neq \Delta_{L_{\mathbf{B}}(\Gamma)}$ be a congruence on $L_{\mathbf{B}}(\Gamma)$. Then, by Proposition $4.3, \rho$ is generated by $\rho_{\text {real }}:=\rho \cap\left(L_{\mathbf{B}}(\Gamma)_{\text {real }}\right)^{2}$ and $\rho_{\text {real }} \neq \Delta_{L_{\mathbf{B}}(\Gamma)_{\text {real }}}$. Hence, there exist two elements $a, b \in L_{\mathbf{B}}(\Gamma)_{\text {real }}$ such that $a \neq b$ and $(a, b) \in \rho$. We claim that there exists a nonzero polynomial $x \in L_{\mathbf{B}}(\Gamma)$ in only real edges such that $(0, x) \in \rho$.

It is clear that $L_{\mathbf{B}}(\Gamma)$ is an additively idempotent hemiring, i.e., $L_{\mathbf{B}}(\Gamma)$ is a partially ordered hemiring with its unique partial order defined as follows: $s \leq s^{\prime} \Longleftrightarrow s+s^{\prime}=s^{\prime}$. Whence, $(a, a+b)=(a+a, a+b) \in \rho,(b, a+b)=$ $(b+b, a+b) \in \rho$, and since $a \neq b$, either $a<a+b$ or $b<a+b$. Thus, keeping in mind that $(a+x, b+x) \in \rho$ for all $x \in L_{\mathbf{B}}(\Gamma)$ and without loss of generality, one may assume that $a<a+b$ and $a, a+b$ are written in the form

$$
a=p_{1}+\cdots+p_{n}, \quad a+b=p_{1}+\cdots+p_{n}+p,
$$

where $p_{1}, \ldots, p_{n}, p$ are distinct paths in $\Gamma$. Moreover, we may choose $a$ having the minimal number $n$ of such $\left\{p_{1}, \ldots, p_{n}\right\}$.

Let $v:=s(p), w:=r(p) \in V$. Then (vaw, $v(a+b) w) \in \rho$, where $v a w=v p_{1} w+\cdots+v p_{n} w$ and $v b w=v p w=p$, hence by minimality we may assume that $s\left(p_{i}\right)=v$ and $r\left(p_{i}\right)=w$ for all $i=1, \ldots, n$.

Suppose that $v \neq w$. Write $p=q p^{\prime}$, where $q$ is a path from $v$ to $w$ of shortest length and $p^{\prime}$ is a closed path based at $w$. Taking into account Remark 2.7, for every $p_{j}$ such that $q^{*} p_{j} \neq 0$ we have $p_{j}=q p_{j}^{\prime}$ for some closed path $p_{j}^{\prime}$ based at $w$. Then we have $\left(q^{*} a, q^{*}(a+b)\right)=\left(q^{*} p_{1}+\cdots+\right.$ $\left.q^{*} p_{n}, q^{*} p_{1}+\cdots+q^{*} p_{n}+q^{*} p\right)=\left(\sum_{j} p_{j}^{\prime}, \sum_{j} p_{j}^{\prime}+p^{\prime}\right) \in \rho$. Therefore, without loss of generality, we may assume that $v=w$, i.e., $p, p_{1}, \ldots, p_{n}$ are distinct closed paths based at $v$, and consider the following two possible cases.

Case 1. There exists exactly one closed simple path based at $v$, say $c:=e_{1} \ldots e_{m}$. It follows that $c$ is actually a cycle, and by condition (2), $c$ has 
an exit $f$, i.e., there exists $j \in\{1, \ldots, m\}$ such that $e_{j} \neq f$ and $s(f)=s\left(e_{j}\right)$. Then, there are some distinct positive integers $k, k_{i}, i=1, \ldots, n$, such that $p=c^{k}$ and $p_{i}=c^{k_{i}}, i=1, \ldots, n$, and let

$$
\begin{gathered}
x:=\left(c^{*}\right)^{k} a=\left(c^{*}\right)^{h_{1}}+\cdots+\left(c^{*}\right)^{h_{r}}+c^{h_{r+1}}+\cdots+c^{h_{n}} \\
y:=\left(c^{*}\right)^{k}(a+b)=\left(c^{*}\right)^{h_{1}}+\cdots+\left(c^{*}\right)^{h_{r}}+c^{h_{r+1}}+\cdots+c^{h_{n}}+v .
\end{gathered}
$$

Obviously, $(x, y) \in \rho$, and therefore, $(0, r(f))=\left(z^{*} x z, z^{*} y z\right) \in \rho$ for $z:=$ $e_{1} \ldots e_{j-1} f$.

Case 2. There exist at least two distinct closed simple paths based at $v$, say $c$ and $d$, and we have $c^{*} d=0=d^{*} c$ by Remark 2.7. Note that $\left(p^{*} a, p^{*}(a+b)\right) \in \rho$ and let

$$
\begin{gathered}
x:=p^{*} a=q_{1}^{*}+\cdots+q_{s}^{*}+q_{s+1}+\cdots+q_{n} \\
y:=p^{*}(a+b)=q_{1}^{*}+\cdots+q_{s}^{*}+q_{s+1}+\cdots+q_{n}+v
\end{gathered}
$$

where $q_{1}, \ldots, q_{n}$ are closed paths in $\Gamma$ based at $v$. Then for some $k \in \mathbb{N}$, where $\left|c^{k}\right|>\max \left\{\left|q_{1}\right|, \ldots,\left|q_{n}\right|\right\}$, we get $x^{\prime}:=\left(c^{*}\right)^{k} x c^{k}=\left(c^{*}\right)^{k} q_{1}^{*} c^{k}+\cdots+$ $\left(c^{*}\right)^{k} q_{s}^{*} c^{k}+\left(c^{*}\right)^{k} q_{s+1} c^{k}+\cdots+\left(c^{*}\right)^{k} q_{n} c^{k}$ and $y^{\prime}:=\left(c^{*}\right)^{k} y c^{k}=\left(c^{*}\right)^{k} q_{1}^{*} c^{k}+\cdots+$ $\left(c^{*}\right)^{k} q_{s}^{*} c^{k}+\left(c^{*}\right)^{k} q_{s+1} c^{k}+\cdots+\left(c^{*}\right)^{k} q_{n} c^{k}+v$, and $\left(x^{\prime}, y^{\prime}\right) \in \rho$. If $\left(c^{*}\right)^{k} q_{i}^{*} c^{k}=0=$ $\left(c^{*}\right)^{k} q_{j} c^{k}$ for all $i=1, \ldots, s$ and $j=s+1, \ldots, n$, then $(0, v)=\left(x^{\prime}, y^{\prime}\right) \in \rho$. Note that if $\left(c^{*}\right)^{k} q_{j} c^{k} \neq 0$, then $\left(c^{*}\right)^{k} q_{j} \neq 0$, and as $\left|c^{k}\right|>\left|q_{j}\right|, c^{k}=q_{j} q_{j}^{\prime}$ for some closed path $q_{j}^{\prime}$ based at $v$. Whence, $q_{j}=c^{r}$ for some positive integer $r \leq k$. Similarly, in the case $\left(c^{*}\right)^{k} q_{i}^{*} c^{k} \neq 0$, we get that $q_{i}^{*}=\left(c^{*}\right)^{s}$ for some positive integer $s \leq k$. Since $c^{*} d=0=d^{*} c$, for every $i, j$, one gets $d^{*}\left(c^{*}\right)^{k} q_{i}^{*} c^{k} d=0=d^{*}\left(c^{*}\right)^{k} q_{j} c^{k} d$, and hence, $(0, v)=\left(d^{*} x^{\prime} d, d^{*} y^{\prime} d\right) \in \rho$.

Finally, let us consider the ideal of $L_{\mathbf{B}}(\Gamma)$ defined as follows:

$$
I:=\left\{x \in L_{\mathbf{B}}(\Gamma) \mid(0, x) \in \rho\right\} .
$$

From the observations above, $I$ contains a nonzero polynomial in only real edges. By our assumption and Theorem 3.4, $L_{\mathbf{B}}(\Gamma)$ is an ideal-simple hemiring, and hence, $I=L_{\mathbf{B}}(\Gamma)$. It immediately follows that $\rho=L_{\mathbf{B}}(\Gamma)^{2}$, which ends the proof.

Combining Proposition 4.1, Theorem 4.4 and [1, Theorem 3.11], we obtain a complete characterization of the congruence-simple Leavitt path algebras $L_{S}(\Gamma)$ of row-finite graphs $\Gamma$ over commutative semirings.

Theorem 4.5. A Leavitt path algebra $L_{S}(\Gamma)$ of a row-finite graph $\Gamma=$ $(V, E, s, r)$ with coefficients in a commutative semiring $S$ is congruencesimple if and only if the following three conditions are satisfied:

(1) $S$ is either a field, or the Boolean semifield $\mathbf{B}$;

(2) The only hereditary and saturated subset of $V$ are $\varnothing$ and $V$;

(3) Every cycle in $\Gamma$ has an exit. 
In light of [2, Theorem 3.1], [23, Theorem 6.18] and [12, Theorem 3.11], and to stimulate an interest of some potential readers in research in this, in our view, quite interesting and promising direction, we post the following

Conjecture. Theorem 4.5 is true for the Leavitt path algebras $L_{S}(\Gamma)$ over an arbitrary graph $\Gamma$.

As was done in the previous section, we end this section and the paper by re-establishing the congruence-simpleness of the Leavitt path algebras of Examples 2.3.

Examples 4.6 ( $c f$. [1, Corollary 3.13]). We can re-establish the congruencesimpleness of the algebras given in Example 3.2 above.

(i) By $\left[15\right.$, Corollary 4.8], $M_{n}(\mathbf{B})$ is congruence-simple. However, this fact can be also justified by Theorem 4.5, since it is easy to check that the graph $\Gamma$ of Examples 2.3 (i) satisfies (1) and (2) of Theorem 4.5.

(ii) By Examples 2.3 (ii), the Laurent polynomial algebra $\mathbf{B}\left[x, x^{-1}\right] \cong$ $L_{S}(\Gamma)$ where the graph $\Gamma$ contains a cycle without an exit, and therefore, by Theorem $4.5, \mathbf{B}\left[x, x^{-1}\right]$ is not congruence-simple.

(iii) By Examples 2.3 (iii), the Leavitt algebras $L_{\mathbf{B}}(1, n)$ for $n \geq 2$ are isomorphic to the Leavitt path algebras $L_{\mathbf{B}}(\Gamma)$ such that for the graphs $\Gamma$ conditions (1) and (2) of Theorem 4.5 are obviously satisfied, and therefore, the algebras $L_{\mathbf{B}}(\Gamma)$ are congruence-simple.

\section{References}

[1] G. Abrams and G. Aranda Pino, The Leavitt path algebra of a graph, Journal of Algebra, 293 (2005), 319-334.

[2] G. Abrams and G. Aranda Pino, The Leavitt path algebras of arbitrary graphs, Houston Journal of Mathematics, 34 (2008), 423-442.

[3] G. Abrams, Leavitt path algebras: the first decade, Bull. Math. Sci., 5 (2015), 59-120.

[4] P. Ara, M. A. Moreno, E. Pardo, Nonstable K-theory for graph algebras, Algebr. Represent. Theory, 10 (2007), no. 2, 157-178.

[5] R. El Bashir, J. Hurt, A. Jančařík, and T. Kepka, Simple commutative semirings, J. Algebra, 236 (2001), 277-306.

[6] R. El Bashir, T. Kepka, Congruence-simple semirings, Semigroup Forum, 75 (2007), 588-608.

[7] G. M. Bergman, Coproducts and some universal ring constructions, Trans. Amer. Math. Soc., 200 (1974), 33-88.

[8] P. Colak, Two-sided ideals in Leavitt path algebras, J. Algebra Appl., 10 (2011), 801-809. 
[9] J. Cuntz, Simple C*-algebras generated by isometries, Comm. Math. Phys., 57 (1977), 173-185.

[10] K. Głazek, A Guide to the Literature on Semirings and their Applications in Mathematics and Information Science, Kluwer Academic Publishers, Dordrecht-Boston-London, 2001.

[11] J. S. Golan, Semirings and their Applications, Kluwer Academic Publishers, Dordrecht-Boston-London, 1999.

[12] K. R. Goodearl, Leavitt path algebras and direct limits, Contemporary Mathematics, 480 (2009), 165-187.

[13] U. Hebisch, H.-J. Weinert, On the rank of semimodules over semirings, Collectanea Mathematica, 46: 1-2 (1998), 83-95.

[14] J. Ježek, T. Kepka, M. Maróti, The endomorphism semiring of a semilattice, Semigroup Forum, 78 (2009), 21-26.

[15] Y. Katsov, T. G. Nam, N. X. Tuyen, More on subtractive semirings: simpleness, perfectness and related problems, Comm. Algebra, 39 (2011), 43424356.

[16] Y. Katsov, T. G. Nam, J. Zumbrägel, On simpleness of semirings and complete semirings, J. Algebra Appl., 13: 6 (2014).

[17] A. Kendziorra and J. Zumbrägel, Finite simple additively idempotent semirings, J. Algebra, 388 (2013), 43-64.

[18] W. G. Leavitt, The module type of a ring, Trans. Amer. Math. Soc., 42 (1962), 113-130.

[19] W. G. Leavitt, The module type of homomorphic images, Duke Math. J., 32 (1965), 305-311.

[20] G. Maze, C. Monico, J. Rosenthal, Public key cryptography based on semigroup actions, Adv. Math. Commun., 1 (2007), 489-507.

[21] S. S. Mitchell and P. B. Fenoglio, Congruence-free commutative seimirings, Semigroup Forum, 31 (1988), 79-91.

[22] C. Monico, On finite congruence-simple semirings, J. Algebra, 271 (2004), 846-854.

[23] M. Tomforde, Uniqueness theorems and ideal structure for Leavitt path algebras, J. Algebra, 318 (2007), 270-299.

[24] M. Tomforde, Leavitt path algebras with coefficients in a commutative ring, Journal of Pure and Applied in Algebra, 215 (2011), 471-484.

[25] J. Zumbrägel, Classification of finite congruence-simple semirings with zero, J. Algebra Appl., 7 (2008), 363-377. 